\title{
Indirect effects of COVID-19 on child health care: delayed diagnosis of developmental dysplasia of the hip
}

\author{
Danilo Buonsenso ${ }^{1,2}\left(\right.$ Nicoletta Menzella $^{1} \cdot$ Rosa Morello $^{1} \cdot$ Piero Valentini $^{1}$
}

Received: 5 June 2020 / Accepted: 23 June 2020 / Published online: 2 July 2020

(c) Società Italiana di Ultrasonologia in Medicina e Biologia (SIUMB) 2020

Developmental dysplasia of the hip (DDH) is a condition that includes a wide spectrum of conditions, from mild hip developmental abnormalities to frank dislocation, which, if untreated, may cause significant long-term complications [1].

Screening by ultrasound is well established as a critical element in the evaluation of the infant hip [2]. Although there are still controversies, in several countries universal ultrasound screening is endorsed, since the procedure is free of risks and has been able to detect DDH in some children with normal clinical examination and no risk factors (which would have been missed, therefore, by the only clinical examination) [3].

In the context of the COVID-19 pandemic, several Institutions decided to defer non-urgent outpatient evaluations to reduce the risk of intra-hospital spread of SARS-CoV-2. However, experts in the DDH field suggested that the screening should not be considered as a non-urgent practice since delayed diagnosis, and treatment can have long-term consequences on child health, and heavy direct and indirect costs (including psychological effects) [1]. While good outcomes may, of course, still be achieved for children who present late with DDH, early detection and treatment is associated with the best results, including the avoidance of surgery. However, due to shortage of human resources and personal protective equipment [4], the re-asset of outpatient activities has not been easily feasible in several contexts.

During the pandemic period, a 5-month-old female with family history positive for DDH, never evaluated before since he was discharged home after birth, was sent to our

Danilo Buonsenso

danilobuonsenso@gmail.com

1 Department of Woman and Child Health and Public Health, Fondazione Policlinico Universitario A. Gemelli IRCCS, Largo A. Gemelli 8, 00168 Roma, Italia

2 Istituto di Microbiologia, Università Cattolica del Sacro Cuore, Roma, Italia attention from another region, to perform a hip ultrasound. Clinically, the Ortolani-Barlow maneuvers were positive and her hip was dislocatable especially on the right side.

Subsequently, we performed the ultrasound examination according to Graaf [2]. The exam showed dysplasia with the shallow and underdeveloped acetabulum, subluxation with a displacement of the joint with some contact remaining between the articular surfaces and dislocation with a displacement of the joint with no contact between the original articular surfaces (Fig. 1). Therefore, a delayed diagnosis of DDH was performed. Considering the stage of DDH and the child age an $\mathrm{x}$-ray examination was required, showing bilateral hip dislocation and delay of ossification.

In this case, having the child a known risk factor for $\mathrm{DDH}$, a screening ultrasound at 4 weeks of age was needed according to the last guidelines [2]. However, the family was not able to obtain an outpatient clinical visit, nor ultrasound evaluation. The family was only able to perform ultrasound at 5 months of age. If recognized at the proper timing, the infant would have probably benefited from non-invasive correction with the use of a harness or brace. In this case, treatment will be more complicated, including open reduction if closed reduction is not successful, with less predictable results. When diagnosed late, DDH can lead to pain and osteoarthritis by early adulthood, produce a difference in leg length or decreased agility. Even with appropriate treatment, hip deformity and osteoarthritis may develop later in life and require early surgical approach with a hip prosthesis.

Although COVID-19 has been shown to be mild in the majority of cases [5], indirect effects related to the restrictive measures are having significant, not yet established, consequences on primary child health. Vaccination delays or missing of doses has been noticed by the American Academy of Pediatrics, but other primary care interventions are at risk as well. However, paediatricians must be committed in supporting parents in not-missing routine child well-being visits, since delays can cause unpredictable consequences, that would have been easily prevented, as in this case. 
Fig. 1 Hip ultrasound and X-ray of the 5-month-old child with developmental dysplasia of the hip. Right (a) and left (c) hip dislocations seen on ultrasound, and confirmed on X-ray (b)
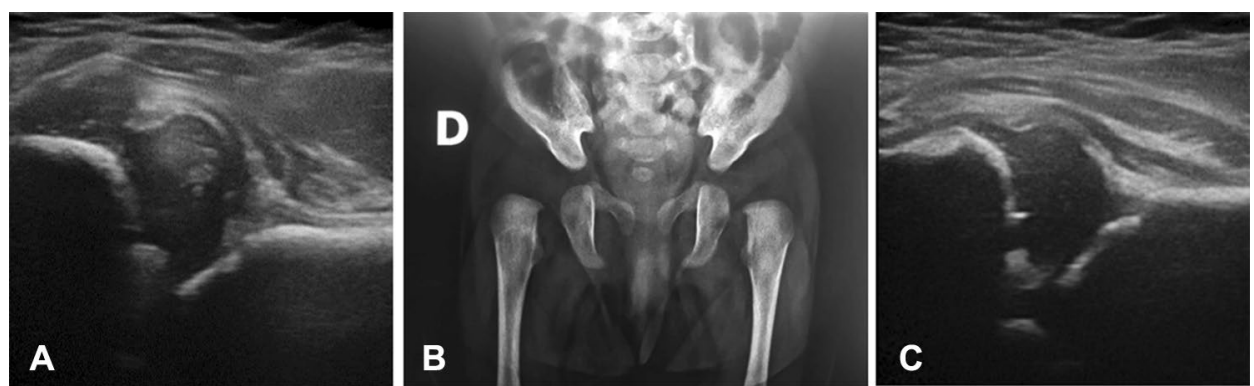

\section{Compliance with ethical standards}

Conflict of interest The authors declare that they have no conflict of interest.

\section{References}

1. O'Beirne J, De Pellegrin M, Casini C, Chlapoutakis K, Aydingoz U, Dubs B, Karantanas A (2020) Developmental dysplasia of the hip in the context of the COVID-19 pandemic. Eur Radiol. https:// www.european-radiology.org/opinions/developmental-dysplasiaof-the-hip-in-the-context-of-the-covid-19-pandemic/. Accessed 27 May 2020.

2. O'Beirne JG, Chlapoutakis K, Alshryda S et al (2019) International interdisciplinary consensus meeting on the evaluation of developmental dysplasia of the hip. Ultraschall Med 40:454-464

3. Buonsenso D, Curatola A, Lazzareschi I et al (2020) Developmental dysplasia of the hip: real world data from a retrospective analysis to evaluate the effectiveness of universal screening. J Ultrasound. https://doi.org/10.1007/s40477-020-00463-w

4. Bressan S, Buonsenso D, Farrugia R et al (2020) Preparedness and response to pediatric COVID-19 in European emergency departments: a survey of the REPEM and PERUKI networks. Ann Emerg Med. https://doi.org/10.1016/j.annemergmed.2020.05.018

5. Parri N, Lenge M, Buonsenso D (2020) Coronavirus infection in pediatric emergency departments (CONFIDENCE) research group. Children with Covid-19 in pediatric emergency departments in Italy [published online ahead of print, 2020 May 1]. N Engl J Med. [NEJMc2007617].

Publisher's Note Springer Nature remains neutral with regard to jurisdictional claims in published maps and institutional affiliations. 